\title{
THE HOST GALAXY OF GRB $990123^{1}$
}

\author{
J. S. Bloom,${ }^{2}$ S. C. Odewahn, ${ }^{2}$ S. G. Djorgovski, ${ }^{2}$ S. R. Kulkarni, ${ }^{2}$ F. A. Harrison, ${ }^{2}$ C. Koresko, ${ }^{2}$ G. Neugebauer, ${ }^{2}$ \\ L. Armus, ${ }^{3}$ D. A. Frail, ${ }^{4}$ R. R. Gal, ${ }^{2}$ R. Sari, ${ }^{2}$ G. Squires, ${ }^{2}$ G. Illingworth, ${ }^{5}$ D. Kelson, ${ }^{6}$ F. H. Chaffee, ${ }^{7}$ \\ R. Goodrich, ${ }^{7}$ M. Feroci, ${ }^{8}$ E. Costa, ${ }^{8}$ L. Piro, ${ }^{8}$ F. Frontera,,${ }^{9,10}$ S. Mao, ${ }^{11}$ \\ C. Akerlof, ${ }^{12}$ AND T. A. MCKAY ${ }^{12}$ \\ Received 1999 February 12; accepted 1999 April 1; published 1999 April 29
}

\begin{abstract}
We present deep images of the field of GRB 990123 obtained in a broadband UV/visible bandpass with the Hubble Space Telescope (HST) and deep near-infrared images obtained with the Keck I $10 \mathrm{~m}$ telescope. The HST image reveals that the optical transient (OT) is offset by 0 ".67 (5.8 kpc in projection) from an extended, apparently interacting galaxy. This galaxy, which we conclude is the host galaxy of GRB 990123, is the most likely source of the absorption lines of metals at a redshift of $z=1.6$ seen in the spectrum of the OT. With magnitudes of Gunn- $r=24.5 \pm 0.2$ and $K=22.1 \pm 0.3 \mathrm{mag}$, this corresponds to an $L \sim 0.5 L_{*}$ galaxy, assuming that it is located at $z=1.6$. The estimated unobscured star formation rate is $\approx 4 M_{\odot} \mathrm{yr}^{-1}$, which is typical for normal galaxies at comparable redshifts. There is no evidence for strong gravitational lensing magnification of this burst, and some alternative explanation for its remarkable energetics (such as beaming) may therefore be required. The observed offset of the OT from the nominal host center, the absence of broad absorption lines in the afterglow spectrum, and the relatively blue continuum of the host do not support the notion that gamma-ray bursts (GRBs) originate from active galactic nuclei or massive black holes. Rather, the data are consistent with models of GRBs that involve the death and/or merger of massive stars. Indeed, the HST image suggests an intimate connection between GRB 990123 and a star-forming region.
\end{abstract}

Subject headings: cosmology: miscellaneous — cosmology: observations — gamma rays: bursts

\section{INTRODUCTION}

Following the detection of GRB 990123 by BeppoSAX (Piro 1999a), we discovered an optical transient (OT) (Odewahn, Bloom, \& Kulkarni 1999) and subsequently a coincident radio transient (Frail \& Kulkarni 1999) within the error circles of the gamma-ray burst (GRB) and the associated fading X-ray source (Piro 1999b). Examination of the ROTSE (Robotic Optical Transient Search Experiment) images taken during the GRB itself revealed a hitherto unseen bright $(m \approx 8.9$ mag at peak) phase of the optical afterglow (Akerlof et al. 1999).

In Kulkarni et al. (1999), we present a comprehensive study of the optical and infrared observations of the transient afterglow and report a measurement of an absorption redshift of $z_{\text {abs }}=1.6$. Combining the redshift with the observed fluence

\footnotetext{
${ }^{1}$ Partially based on the observations obtained at the W. M. Keck Observatory which is operated by the California Association for Research in Astronomy, a scientific partnership among California Institute of Technology, the University of California, and the National Aeronautics and Space Administration.

${ }^{2}$ Palomar Observatory, Caltech 105-24, Pasadena, CA 91125.

${ }^{3}$ Infrared Processing and Analysis Center, Caltech, MS 100-22, 770 S. Wilson Avenue, Pasadena, CA 91125.

${ }^{4}$ National Radio Astronomy Observatory, P.O. Box O, 1003 Lopezville Road, Socorro, NM 87801.

${ }^{5}$ University of California Observatories/Lick Observatory, University of California at Santa Cruz, Santa Cruz, CA 95064.

${ }^{6}$ Department of Terrestrial Magnetism, Carnegie Institute of Washington, 5241 Broad Branch Road, NW, Washington, DC 20015-1305.

${ }^{7}$ W. M. Keck Observatory, 65-0120 Mamalahoa Highway, Kamuela, HI 96743.

${ }^{8}$ Instituto di Astrofisica Spaziale CNR, via Fosso del Cavaliere, Roma, I-00133, Italy.

${ }^{9}$ ITESRE-CNR, Via Gobetti 101, Bologna, I-40129, Italy.

${ }^{10}$ Physics Department, University of Ferrara, Via Paradiso 12, Ferrara, I-44100, Italy.

${ }^{11}$ Max-Planck-Institut für Astrophysik, Karl-Schwarzschild-Strasse 1, Postfach 1523, Garching, 85740, Germany.

${ }^{12}$ Department of Physics, University of Michigan at Ann Arbor, 2071 Randall, Ann Arbor, MI 48109-1120.
}

(Feroci et al. 1999) results in an inferred energy release of $3 \times 10^{54}$ ergs (if the emission was isotropic), which clearly poses a problem to most conventional models of GRBs. However, noting a break in the optical afterglow decay, Kulkarni et al. (1999) argue that the emission geometry may have been jetlike; this would then decrease the energy constraint.

Both currently favored models of GRB progenitors, the death of a massive star (Woosley 1993; Paczyński 1998) and the coalescence of a neutron star (NS) or NS-black hole $(\mathrm{BH})$ binary (Paczyński 1986; Goodman 1986; Narayan, Paczyński, \& Piran 1992), predict that GRB rates should correlate strongly with the cosmic star formation rate (SFR), and so most GRBs should occur during epochs of the highest SFR (i.e., a redshift range of $z=1-3$ ). The former model predicts a tight spatial correlation between GRBs and star-forming regions in galactic disks. The latter, however, allows the coalescence site of a merging binary component to be quite distant (beyond a few kiloparsecs) from the stellar birth site (Bloom, Sigurdsson, \& Pols 1999). GRBs could also be associated with nuclear black holes (i.e., active galactic nuclei) (see Roland, Frossati, \& Teyssier 1994). In this scenario, unlike either model described above, the GRBs will preferentially occur in the center of the host. The exquisite angular resolution of the Hubble Space Telescope (HST) is well suited to address this issue of the locations of GRBs relative to the host galaxies. In this Letter, we report on HST observations of the host galaxy of GRB 990123 taken about 16 days after the burst as well as Keck imaging in the near-infrared.

\section{OBSERVATIONS AND DATA REDUCTIONS}

The ground-based near-IR images of the field of GRB 990123 were obtained using the near-infrared camera (Matthews \& Soifer 1994) on the Keck I 10 m telescope. A log of the observations and a detailed description of the data and the 


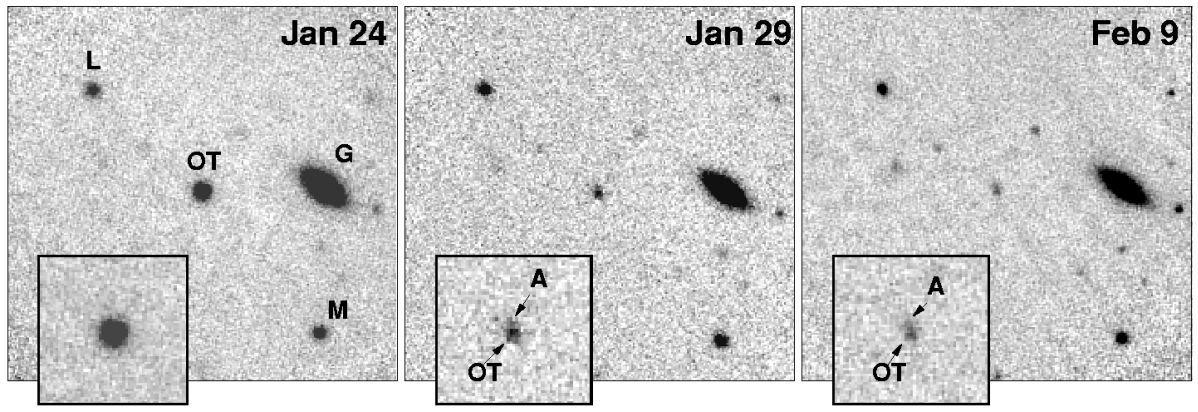

FIG. 1.-Three epochs of Keck I $K$-band imaging of the field of GRB 990123 (1999 January 24.6, January 29.7, and February 9.6 UT). The field shown is $32^{\prime \prime} \times 32^{\prime \prime}$, corresponding to about $270 \times 270$ physical kpc in projection at $z=1.6004$ (for $H_{0}=65 \mathrm{~km} \mathrm{~s}^{-1} \mathrm{Mpc}^{-1}$ and $\Omega_{0}=0.2$ ). The images have been rotated to the standard orientation, so that the east is to the left and north is up. The magnitude of the host galaxy is $K_{\text {host }}=22.1 \pm 0.3$ mag. In the January 24 image, the OT dominates the host galaxy flux $\left(K_{\text {От }}=18.29 \pm 0.04\right.$; Kulkarni et al. 1999), but by January 29 the galaxy is resolved (see inset) from the OT.

reduction procedures are given by Kulkarni et al. (1999). The observations were obtained in the $K$ or $K_{s}$ bands and were calibrated to the standard $K$ band (effective wavelength $=2.195$ $\mu \mathrm{m})$. The Galactic extinction correction is negligible in the $K$ band (see below).

The first evidence of the underlying galaxy, approximately 0 ".6 from the OT, was seen in our Keck $K$-band images taken on 1999 January 27 UT. The galaxy, the putative host (which we designate as "A"), was then clearly detected in the images obtained on 1999 January 29 UT (Djorgovski et al. 1999a) and later, under excellent seeing conditions, on February 9 and 10 (see Fig. 1). Sets of pixels dominated by the OT or by the galaxy were masked, and total fluxes with such censored data were evaluated in photometric apertures of varying radii. Total fluxes of the OT + galaxy were also measured in the same apertures using the uncensored data. We also varied the aperture radii, and the position and the size of the sky measurement annulus. On February 9 (February 10) UT, we found that the OT contributes $65 \%(57 \%)( \pm 10 \%)$ of the total OT + galaxy light. The estimated errors of the fractional contributions of the OT to the total light reflect the scatter obtained from variations in the parameters of these image decompositions. In both epochs, the fractional contribution of the host implies that a flux of the host galaxy is $0.9 \pm 0.3 \mu \mathrm{Jy}\left(K_{\text {host }}=22.1 \pm 0.3 \mathrm{mag}\right)$. We assume $636 \mathrm{Jy}$ for the flux zero point of the $K$ band for $K=0$ mag (Bessel \& Brett 1988).

The HST observations of the GRB 990123 field were obtained in 1999 February 8-9 UT in response to the director's discretionary time proposal GO-8394, with the immediate data release to the general community (Beckwith 1999). The CCD camera of the Space Telescope Imaging Spectrograph (STIS) (Kimble et al. 1998) in CLEAR aperture (50CCD) mode was used. Over the course of three orbits, the field was imaged in six positions dithered in a spiral pattern for a total integration time of $7200 \mathrm{~s}$. Each position was imaged twice to facilitate cosmic-ray removal (adding to a total of 12 integrations).

Initial data processing followed the STScI pipeline procedures, including bias and dark current subtraction. The six cosmic-ray-removed images were then combined by registering the images and median-stacking to produce a master science-grade image. We also produced a higher resolution image using the "drizzle" technique (Fruchter \& Hook 1999). Photometry and astrometry were performed on both final image products. We find a negligible difference between the two images compared with other uncertainties (e.g., sky determination and counting noise) in photometry and astrometry. The mean epoch of the final images is 1999 February 9.052 UT.
Figure 2 shows a portion of the STIS image of the GRB 990123 field. We find (see below) the OT clearly detected as a point source $0.67 \pm 0.02$ to the southwest of the central region of galaxy A. Galaxy A has an elongated and clumpy appearance, possibly indicative of star formation regions in a late-type galaxy. A morphological classification as an irregular galaxy or interacting galaxy system is likely most apt. Such morphologies are typical for many galaxies at comparable flux levels, as observed with the HST. Its extension to the south clearly overlaps with the OT, and it is thus very likely that this galaxy is responsible for the absorption-line system at $z_{\mathrm{abs}}=$ 1.6004 (Kulkarni et al. 1999; Hjorth et al. 1999). The knot "B" to the east may be a satellite of the host galaxy or a star-forming region along the interface zone of a galaxy interacting with the host.

We measured the centroid of the optical transient in our discovery image from January 23 at the Palomar 60 inch telescope (P60). The OT was bright $(r=18.65)$ at this early epoch, and its position is well determined with respect to other objects in the field. Next we computed the astrometric mapping of the P60 coordinate system to a deep Keck II $10 \mathrm{~m} R$-band image from 1999 February 9.6 UT (Kulkarni et al. 1999) using 75 well-detected objects common to the two images. Similarly, we tied the Keck II coordinates to the STIS image using 19 common tie objects. We found the ground-based position of the OT to be consistent with the STIS point source, with a negligible offset of $00^{\prime \prime} 09 \pm 00^{\prime \prime} 18$.

The coordinates of the OT as measured in the HST image are $\alpha=15^{\mathrm{h}} 25^{\mathrm{m}} 30^{\mathrm{s}} 3026, \delta=+44^{\circ} 45^{\prime} 59^{\prime \prime} .048(\mathrm{~J} 2000)$; the HST plate solution is based on the revised guide star catalog and is accurate (in an absolute sense) to $\sim 0^{\prime \prime} .3$. We note the excellent agreement between this HST measurement and the absolute astrometric measurement from ground-based imaging as reported in Kulkarni et al. (1999). The brightest central region of galaxy A (itself extended north-south) is located 0".17 east, 0 "64 north of the transient. The bright, possibly star-forming, regions "A1" and "B" are located 0"25 west, 0".46 north and 0 ".66 east, 0".51 south, respectively. The uncertainties in the relative positions are $\sim 20$ mas.

No other galaxies brighter than $V \sim 27$ mag are detected in the STIS image closer to the OT than galaxy A, and we see no evidence for a distant cluster (or even a sizable group) in this field. This effectively removes the possibility (Djorgovski et al. 1999b) that the burst was significantly magnified by gravitational lensing.

We will assume for the Galactic reddening in this direction $E_{B-V}=0.016$ mag (Schlegel, Finkbeiner, \& Davis 1998), and 

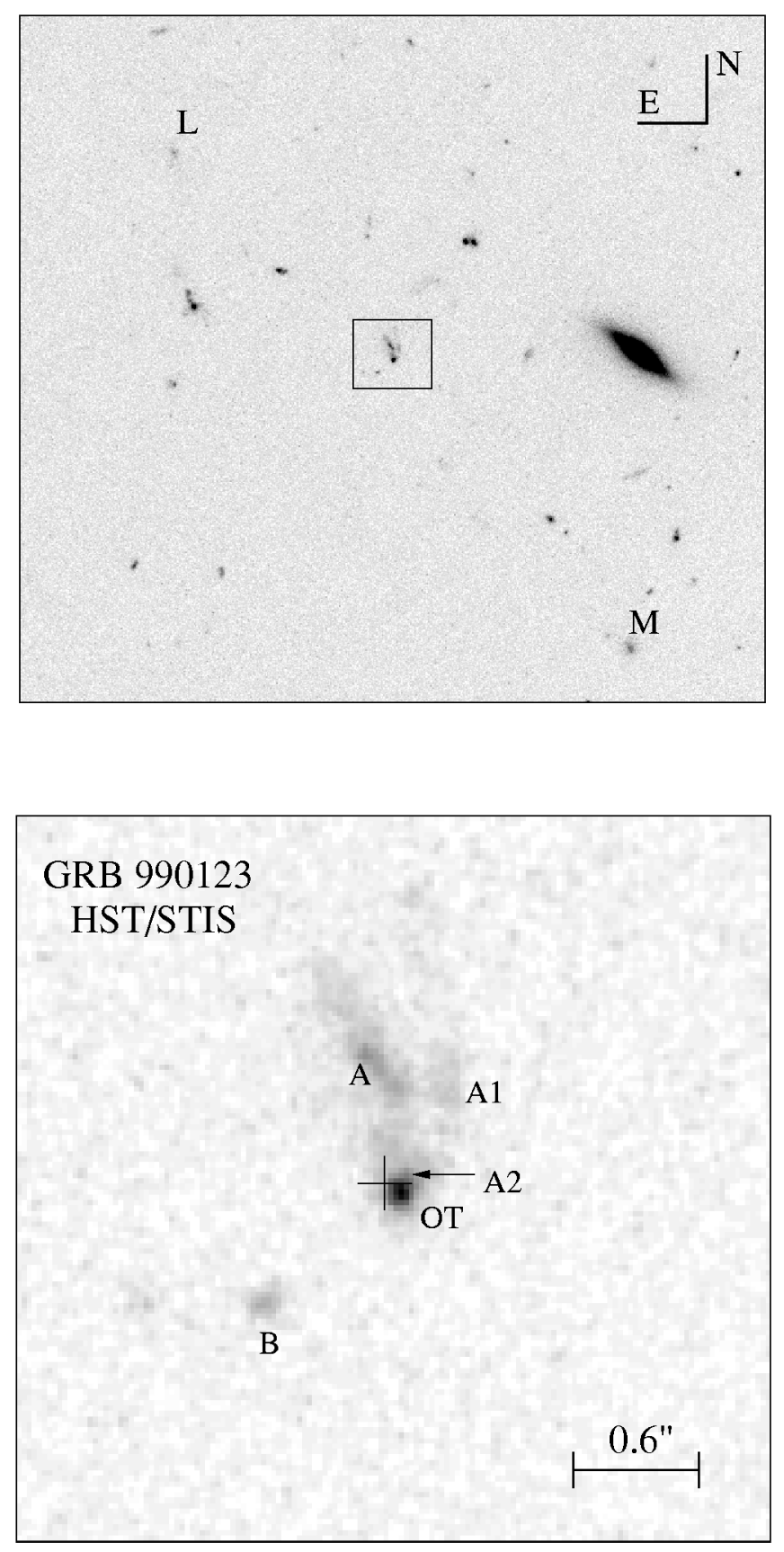

FIG. 2.-The HST STIS "drizzle" image (mean epoch 1999 February 9.052 UT) of the field of GRB 990123 rotated to the normal orientation. Top: The field shown is $32 \times 32 \operatorname{arcsec}^{2}$, corresponding to about $270 \times 270$ proper kpc ${ }^{2}$ $\left(710 \times 710\right.$ comoving $\mathrm{kpc}^{2}$ ) in projection at $z=1.6$ (for $H_{0}=65 \mathrm{~km} \mathrm{~s}^{-1}$ $\mathrm{Mpc}^{-1}$ and $\Omega_{0}=0.2$ ). The effective exposure time is $7200 \mathrm{~s}$, and the pixel scale is 0.0254 pixel $^{-1}$. Bottom: The OT, galaxy A (the putative host), and the bright knots (A1 and B) associated with galaxy A are denoted. The cross that overlays the OT point source depicts the $1 \sigma$ uncertainty in each axis for the position of the OT as measured in ground-based imaging (see text). The positional consistency definitively establishes that the point source is indeed the OT. The nebulosity (A2) just to the north of the OT may be a star-forming region (see also Holland \& Hjorth 1999).

we will use the standard Galactic extinction curve with $R_{V}=$ $A_{V} / E_{B-V}=3.1$ to estimate extinction corrections at other wavelengths. We assume the photometric flux zero points as tabulated by Fukugita, Shimasaku, \& Ichikawa (1995).

Most objects detected in the STIS imaging are also detected in the deep Keck II image. After photometric transformation of the $R$-band Keck II image to the Gunn- $r$ system, we used objects common to both images in order to find a zero point for the STIS image. The rms uncertainty of the zero point is $0.1 \mathrm{mag}$, which is mostly due to varied color terms. This scatter implies a transformation of the broadband STIS magnitudes to the Gunn- $r$ system that is rather robust. Taking the system as a whole, $r_{\mathrm{OT}+\text { host }}=24.1 \pm 0.1$, which is in excellent agreement with the Keck II imaging (Kulkarni et al. 1999) taken $12 \mathrm{hr}$ before the HST imaging. Aperture photometry on the individual components yields $r_{\mathrm{OT}}=25.3 \pm 0.2$ and $r_{\text {host }}=24.5 \pm 0.2$ $\left(F_{\nu, r, \text { host }}=0.5 \pm 0.1 \mu \mathrm{Jy}\right)$. The errors are dominated by uncertainties in the color term of the object and the sky value for aperture photometry. Since some flux from the host galaxy must contribute some flux in the OT aperture, the magnitude presented above should be considered an upper (lower) limit for the host (OT).

We also determined the OT and host magnitudes by converting observed counts to flux given the instrumental response. Since the bandpass of the STIS CLEAR is so broad, the conversion depends on the assumed spectrum of the object. For the OT, we assumed a spectral index of $\beta \approx-0.8$ (with $F_{\nu} \sim \nu^{\beta}$ ), as inferred from theory and other OTs. For the host, we take $\beta=-0.5$ as a good approximation for star-forming galaxies in the redshift range of the host, which is consistent with the $K$-band measurement. In both cases, we explored a range of plausible spectral indices. Using the STIS exposure simulator available from STScI to convert the observed counts to flux, we find $V_{\text {От }}=25.4$ and $V_{\text {host }}=24.6$. In a recent paper, Fruchter et al. (1999b), from analysis of the same HST data, find $V_{\text {От }}=25.45 \pm 0.15$ and $V_{\text {host }}=24.20 \pm 0.15$. The determination of the host magnitude is subject to two systematic uncertainties: the assumed galaxy spectrum and the aperture employed in photometry (see above). Within these errors, these measurements are in agreement. Given the assumed spectral shapes, we also find $r_{\mathrm{OT}}=25.1$ and $r_{\text {host }}=24.3$, in agreement with our direct photometric tie to ground-based imaging.

We note that the simple power-law approximation to the broadband spectrum of the galaxy, as defined by our STIS and $K$-band measurements, is $\beta_{\text {host }} \approx-0.5$. This relatively blue color is suggestive of active star formation, but it cannot be used to estimate the SFR directly.

\section{DISCUSSION}

In what follows, we assume a standard Friedman model cosmology with $H_{0}=65 \mathrm{~km} \mathrm{~s}^{-1} \mathrm{Mpc}^{-1}, \Omega_{0}=0.2$, and $\Lambda_{0}=0$. For $z=1.6004$, the luminosity distance is $3.7 \times 10^{28} \mathrm{~cm}$, and 1 " corresponds to 8.64 proper $\mathrm{kpc}$ or 22.45 comoving $\mathrm{kpc}$ in projection.

It is highly likely that the absorption system at $z_{\text {abs }}=$ 1.6004 originates from galaxy $\mathrm{A}$, since no other viable candidate is seen in the $H S T$ images. The proximity of the center of galaxy A to the OT line of sight $\left(0.67 \pm 00^{\prime \prime} 02\right)$, corresponding to 5.8 proper $\mathrm{kpc}$ at this redshift, strongly suggests that the two are physically related. We thus propose that galaxy A is the host galaxy of the GRB. Visual inspection of Figure 2 suggests that a probability of chance superposition at this magnitude level is very small.

In order to estimate the rest-frame luminosity of galaxy A, we interpolate between the observed STIS and $K$-band data points using a power law, to estimate the observed flux at $\lambda_{\text {obs }} \approx 11570 \AA$, corresponding approximately to the effective wavelength of the rest-frame $B$ band. We obtain $F_{v, B \text {, rest }} \approx$ $0.7 \mu \mathrm{Jy}$, corresponding to the absolute magnitude $M_{B}=$ 
-20.0. Locally, an $L_{*}$ galaxy has $M_{B} \approx-20.75$. We thus conclude that this object has the rest-frame luminosity that is $L_{\text {host }} \approx 0.5 L_{*, \text { local }}$. Given the uncertainty of the possible evolutionary histories, it may evolve to become either a normal spiral galaxy or a borderline dwarf galaxy.

We can make a rough estimate of the SFR from the continuum luminosity at $\lambda_{\text {rest }}=2800 \AA$, following Madau, Pozzetti, $\&$ Dickinson (1998). Using the $F_{\nu, 2800}$ estimates given above, the corresponding monochromatic rest-frame power is $P_{\nu, 2800}=2.9 \times 10^{28} \mathrm{ergs} \mathrm{s}^{-1} \mathrm{~Hz}^{-1}$ (for $\beta=0$, since it may be appropriate in the UV continuum itself) or $P_{\nu, 2800}=3.6 \times$ $10^{28} \mathrm{ergs} \mathrm{s}^{-1} \mathrm{~Hz}^{-1}$ (for $\beta=-0.8$ ). The corresponding estimated unobscured SFRs are $\approx 3.6$ and $\approx 4.6 M_{\odot} \mathrm{yr}^{-1}$, probably accurate to within $50 \%$ or better. This modest value is typical for normal galaxies at such redshifts. It is of course a lower limit, since it does not include any extinction corrections in the galaxy itself or any fully obscured star formation.

Further insight into the physical properties of this galaxy comes from its absorption spectrum, presented in Kulkarni et al. (1999). The lines are unusually strong, placing this absorber in the top $10 \%$ of all $\mathrm{Mg}$ II absorbers detected in complete surveys (Steidel \& Sargent 1992). Unfortunately, without a direct measurement of the hydrogen column density, it is impossible to estimate the metallicity of the gas. We note that strong metal-line absorbers are frequently associated with high hydrogen column density systems, such as damped Ly $\alpha$ absorbers. The small scatter of redshift in the individual lines implies a very small velocity dispersion, less than $60 \mathrm{~km} \mathrm{~s}^{-1}$ in the galaxy's rest frame. This implies that the absorber is associated with either a dwarf galaxy or a dynamically cold disk of a more massive system.

The OT is well offset $(5.8 \mathrm{kpc})$ from the central region of the host; this clearly casts doubt on an active galactic nucleus origin of GRBs. However, if the host is indeed an interacting galaxy system, then it is plausible that a massive black hole could be created off-center (as recently suggested by Fruchter et al. 1999b), and thus the position of the GRB 990123 could still permit massive black holes as the progenitors of GRBs. Yet, around such massive BHs, the expectation is that the highvelocity-enshrouding material would give rise to absorption in the GRB afterglow. The clear absence of broad absorption lines in our optical afterglow spectrum, then, does not bode well for the massive black hole hypothesis for the origin of GRBs.
The spatially resolved imaging using HST provides the clearest picture of the relation of GRBs to their hosts. The transient of GRB 970228 is displaced from its host center (Sahu et al. 1997; Fruchter et al. 1999a) but still lies within the half-light radius. GRB 970508, on the other hand, is coincident with the nucleus of its host galaxy to 0"01 (Fruchter \& Pian 1998; Bloom et al. 1998). As shown in this Letter, GRB 990123 is separated from the central region of the host and appears to be spatially coincident with a bright star-forming region ("A2"; see Fig. 2). Indeed, Holland \& Hjorth (1999) have recently corroborated this claim by noting that the size and luminosity of A2 befit the properties of a generic star-forming region. The close connection of GRBs to their hosts can be extended to results from ground-based astrometry by using the host galaxy magnitude as an objective measure (Odewahn et al. 1996) of the host size. Using the total magnitude of all known host galaxies to date, we note that the optical transients (except for GRB 990123) lie well within the effective half-light radius.

Copious star formation always appears to be spatially concentrated: along spiral arms, in bright compact $\mathrm{H}$ II regions in dwarf irregular galaxies, and in the interface zone of interacting galaxies. Given the morphology of the host (Fig. 2), we suggest that GRB 990123 arose from a star-forming complex in the interface zone of what appears to be a pair of interacting galaxies. This is the first clear case of a GRB associated with an interaction region. Another possible case is the host of GRB 980613. Until now, the GRB star formation connection has been primarily through gross star formation rates obtained from spectroscopic indicators. It is possible that with increasingly larger samples of host galaxies, in analogy to supernovae, the relationship of GRBs to the morphology of the hosts may provide complementary insight into the progenitors of GRBs.

We are grateful to S. Beckwith of STScI for the allocation of the director's discretionary time for this project and to the entire BeppoSAX team and the staff of W. M. Keck Observatory for their efforts. We also thank L. Ferrarese for aiding us with HST observing and the anonymous referee for helpful and clarifying comments. This work was supported in part by a grant from STScI, grants from the NSF and NASA, and the Bressler Foundation.

\section{REFERENCES}

Akerlof, C. W., et al. 1999, Nature, 398, 400

Beckwith, S. 1999, GCN Circ. 245 (http://gcn.gsfc.nasa.gov/gcn/gcn3/ 245.gcn3)

Bessell, M. S., \& Brett, J. M. 1988, PASP, 100, 1134

Bloom, J. S., Djorgovski, S., Kulkarni, S. R., \& Frail, D. A. 1998, ApJ, 507, L25

Bloom, J. S., Sigurdsson, S., \& Pols, O. R. 1999, MNRAS, in press (astro$\mathrm{ph} / 9805222$ )

Djorgovski, S. G., et al. 1999a, GCN Circ. 256 (http://gcn.gsfc.nasa.gov/gcn/ gen $3 / 256 . g c n 3)$

Djorgovski, S. G., Kulkarni, S. R., Bloom, J. S., Odewahn, S. C., Gal, R. R., \& Frail, D. A. 1999b, GCN Circ. 216 (http://gcn.gsfc.nasa.gov/gcn/gcn3/ 216.gcn3)

Feroci, M., et al. 1999, IAU Circ. 7095

Frail, D. A., \& Kulkarni, S. R. 1999, GCN Circ. 211 (http://gcn.gsfc.nasa.gov/ $\mathrm{gcn} / \mathrm{gcn} 3 / 211 . \mathrm{gcn} 3)$

Fruchter, A. S., \& Hook, R. N. 1999, PASP, submitted (astro-ph/9808087)

Fruchter, A. S., \& Pian, E. 1998, GCN Circ. 151 (http://gcn.gsfc.nasa.gov/gcn/ $\mathrm{gcn} 3 / 151 . \mathrm{gcn} 3)$

Fruchter, A. S., et al. 1999a, ApJ, 516, 683

1999b, ApJ, submitted (astro-ph/9902236)

Fukugita, M., Shimasaku, K., \& Ichikawa, T. 1995, PASP, 107, 945

Goodman, J. 1986, ApJ, 308, L47
Hjorth, J., Andersen, M. I., Pedersen, H., Zapatero-Osorio, M. R., Perez, E., \& Castro Tirado, A. J. 1999, GCN Circ. 249 (http://gcn.gsfc.nasa.gov/gcn/ $\mathrm{gcn} 3 / 249 . \mathrm{gcn} 3)$

Holland, S., \& Hjorth, J. 1999, A\&A, 344, L67

Kimble, R. A., et al. 1998, ApJ, 492, L83

Kulkarni, S. R., et al. 1999, Nature, 398, 389

Madau, P., Pozzetti, L., \& Dickinson, M. 1998, ApJ, 498, 106

Matthews, K., \& Soifer, B. T. 1994, Infrared Astronomy with Arrays, the Next Generation, ed. I. McLean (Dordrecht: Kluwer), 239

Narayan, R., Paczyński, B., \& Piran, T. 1992, ApJ, 395, L83

Odewahn, S. C., Bloom, J. S., \& Kulkarni, S. R. 1999, IAU Circ. 7094

Odewahn, S. C., Windhorst, R. A., Driver, S. P., \& Keel, W. 1996, ApJ, 472, L13

Paczyński, B. 1986, ApJ, 308, L43 1998, ApJ, 494, L45

Piro, L. 1999a, GCN Circ. 199 (http://gcn.gsfc.nasa.gov/gen/gen3/199.gen3) 1999b, GCN Circ. 203 (http://gcn.gsfc.nasa.gov/gcn/gcn3/203.gcn3)

Roland, J., Frossati, G., \& Teyssier, R. 1994, A\&A, 290, 364

Sahu, K. C., et al. 1997, Nature, 387, 476

Schlegel, D. J., Finkbeiner, D. P., \& Davis, M. 1998, ApJ, 500, 525

Steidel, C. C., \& Sargent, W. L. W. 1992, ApJS, 80, 1

Woosley, S. E. 1993, ApJ, 405, 273 